Müller, Simon; Schreiter, Karolin; Luckner, Robert; Manzey, Dietrich

\title{
Manual flying and energy awareness
}

Beneficial effects of energy displays combined with a new approach of augmented thrust control

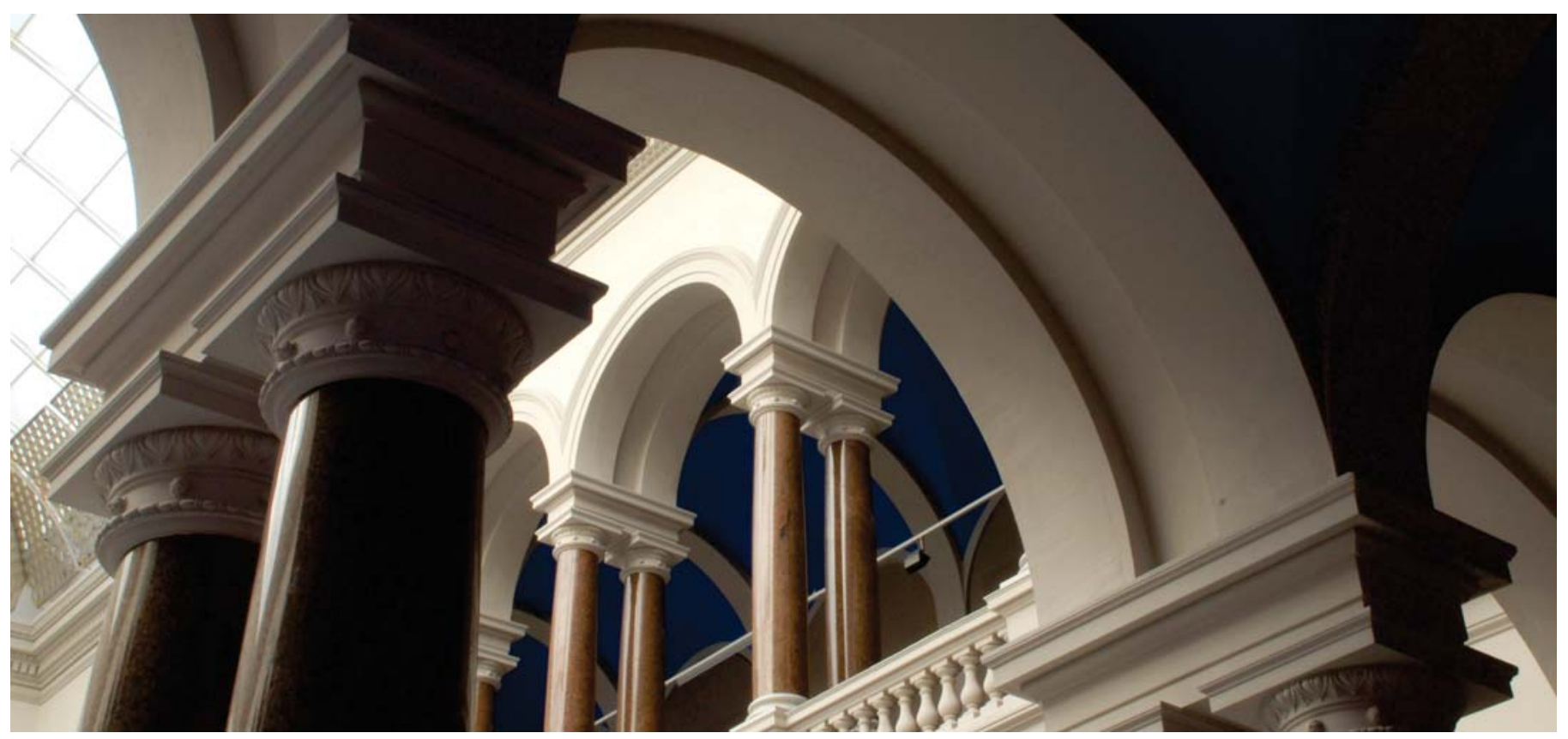

Müller, S.; Schreiter, K.; Luckner, R.; Manzey, D. (2017): Manual Flying and Energy Awareness. Aviation Psychology and Applied Human Factors, 7(1), 18-27. https://doi.org/10.1027/2192-0923/a000111.

Aviation Psychology and Applied Human Factors, 7, 1 ๑ 2017 by Hogrefe Publishing.

This version of the article may not completely replicate the final version published in Aviation Psychology and Applied Human Factors. It is not the version of record and is therefore not suitable for citation. 


\title{
Manual Flying and Energy Awareness
}

\section{Beneficial Effects of Energy Displays Combined With a New Approach of Augmented Thrust Control}

\author{
Simon Müller, ${ }^{1}$ Karolin Schreiter, ${ }^{2}$ Robert Luckner, ${ }^{2}$ and Dietrich Manzey ${ }^{1}$ \\ ${ }^{1}$ Department of Work, Engineering and Organizational Psychology, Technische Universität Berlin, Berlin, Germany \\ ${ }^{2}$ Department of Flight Mechanics, Flight Control and Aeroelasticity, Technische Universität Berlin, Berlin, Germany
}

Manually flying an aircraft can be understood as a complex task of managing physical energies (e.g., Filippone, 2012). In order to accomplish certain objectives like climbing, descending, or level flight, the pilot must take care to provide the aircraft with proper total energy in terms of thrust or drag. Then, the available energy must be distributed across potential energy, representing the altitude of the aircraft, and kinetic energy, representing its speed. This is usually achieved by elevator deflections via control inputs, that is, yoke or sidestick. However, finding appropriate thrust settings and maintaining a proper awareness of the energies needed for a target flight state are not always an easy task.

The complexity of energy management in manual flight can best be illustrated by the control of potential and kinetic energy during approach and landing. The main goal during this flight phase is to fully reduce the total energy of the aircraft until it eventually touches down and stops on the runway. Several ways to reduce energy are available and may be used for this purpose, including lowering the thrust setting and/or inducing additional drag by extending speed brakes, flaps, or landing gears. However, during approach and landing this task is complicated by the fact that the full reduction of energy needs to be accomplished until touchdown at a certain range on a runway. At the same time, steady flight states are repeatedly required throughout the approach. To ensure this, pilots need to continuously gauge both the thrust as well as the aircraft's pitch angle by commanding elevator deflections to control the relative reduction of potential and kinetic energy in an appropriate way. However, the specific settings needed in a given flight situation directly depend on factors such as the actual aircraft mass, configuration of the high lift system, airspeed, and altitude. Thus, the proper thrust and pitch angle settings required for target flight states can change considerably during the flight. Because no pilot can ever memorize all proper pitch angle and thrust settings for a given aircraft and all possible constellations of flight parameters, pilots usually base their control inputs on a subset of crucial combinations that they know. Subsequently, they make adjustments to obtain the proper settings based on a trial-and-error principle. This approach is called pitch-and-power flying and belongs to the basic flying skills of pilots. It generally works well in routine situations of manual flight. But, it can become extremely cognitively demanding, for example, in situations requesting complex changes of energy, in phases of high workload, and in safety-critical conditions. 
Using pitch and power as cue values to control the flight state has evolved historically. But this lacks precise information of the physical energies and does not seem to ensure pilots have sufficient energy awareness, which can even compromise flight safety. This is suggested by several recent incidents and accidents in civil aviation showing that even highly trained pilots can lack essential energy awareness in energy-critical phases of a flight (Dutch Safety Board, 2010; National Transportation Safety Board, 2013). It often results from insufficient monitoring and consideration of all crucial flight parameters in high-workload phases of a flight, but can also be due to an inappropriate mental processing and understanding of the relevant flight parameters with respect to the current flight state of the plane. One way out of this problem is to keep the pilot out of the loop of direct control and let the aircraft always fly and land automatically. However, this is in direct contrast to the current request posed by the Federal Aviation Administration (2013; 2016), to even increase the manual flying in commercial aviation in order to avoid issues of de-skilling of pilots. Moreover, several of the most recent issues of energy awareness and proper energy management occurred when pilots were forced to take over control from the autopilot, that is, in situations where the automation failed for some reason.

Thus, instead of only relying on cockpit automation, measures are needed that better support pilots in finding proper thrust settings and unload them from the typical demands of pitch-and-power flying when flying manually. This is the main objective of a new system referred to as $n x$ Control that has been proposed recently based on a new concept of thrust control and the provision of augmented energy information to the pilot (Müller, Schreiter, Manzey, \& Luckner, 2016). Specifically, it consists of two components. The first component includes a primary flight display (PFD) supplemented by additional energy indicators that inform the pilot about the change of the aircraft's energy in a direct and salient way. The second one includes an assistive demand controller that transfers the concepts of fly-by-wire flight control laws for an aircraft's attitude also to the control of thrust. However, the bandwidth of thrust control is an order of magnitude lower than for attitude control.

The idea of augmented cues providing information about the current energy state of an aircraft is not new. In fact, various ideas and possible implementations of what is referred to as energy displays have already been proposed (e.g., Amelink, Mulder, van Paassen, \& Flach, 2005; Catton, Starr, Noyes, Fisher, \& Tim, 2007; Lambregts, Rademarker, \& Theunissen, 2008). The common element of most of these concepts includes an augmented presentation of important energy status information as integral part

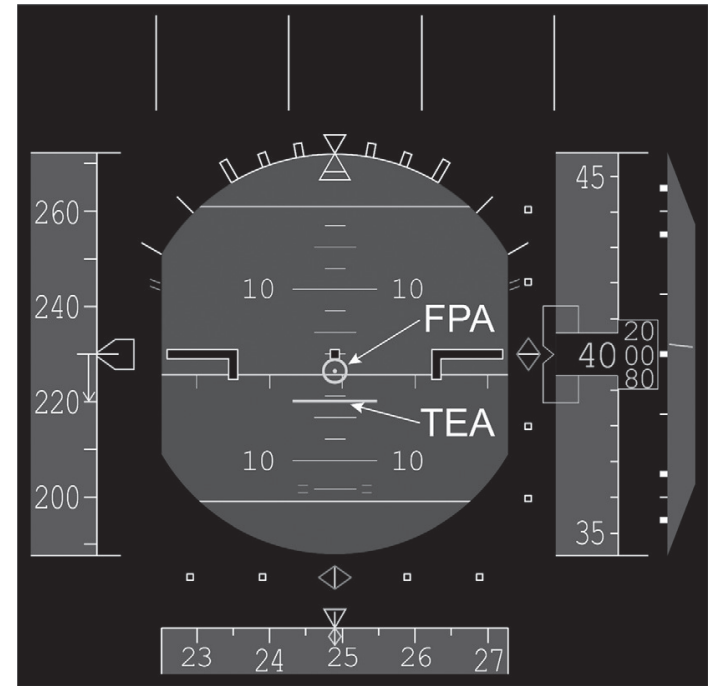

Figure 1. Energy-enhanced primary flight display with FPA and TEA in the center of attitude indicator. FPA = flight path angle; TEA = total energy angle.

of the PFD, mostly represented by indicators of the total energy angle (TEA, also known as potential flight path angle) and the flight path angle (FPA; Amelink et al., 2005; Lambregts et al., 2008). The TEA provides information about the current rate of change of total energy. The FPA provides information about the current rate of change in potential energy. The implementation of these indicators, in the format chosen for the nxControl system, is shown in Figure 1 based on the example of a current Airbus A320 PFD (Müller, Manzey, Schreiter, \& Luckner, 2015). The TEA is represented by a horizontal line; the FPA is shown as a circle with a dot in its center. According to Airbus color standards green color was chosen for both symbols. The main advantage of this display is that the dynamic spatial interaction of both the TEA and FPA provides a direct and integrated picture of the control inputs' consequences on kinetic, potential, and total energy. At the same time, it informs whether the aircraft accelerates or decelerates and climbs or descends at a glance in the following way (see Figure 2): While the relative position of the FPA in relation to the artificial horizon line directly indicates if the aircraft is descending or climbing, the relative position of the TEA and FPA, that is, whether the TEA is above or below the FPA, shows if it is gaining or losing speed. If both symbols converge, it indicates that the aircraft is moving with constant speed. Thus, the specific spatial relation of the TEA, FPA, and artificial horizon line always provides information about the current energy state in what has been referred to as an emergent feature (Bennet \& Flach, 2011). The use of the emergent feature in display design has been proposed in particular to reduce the 


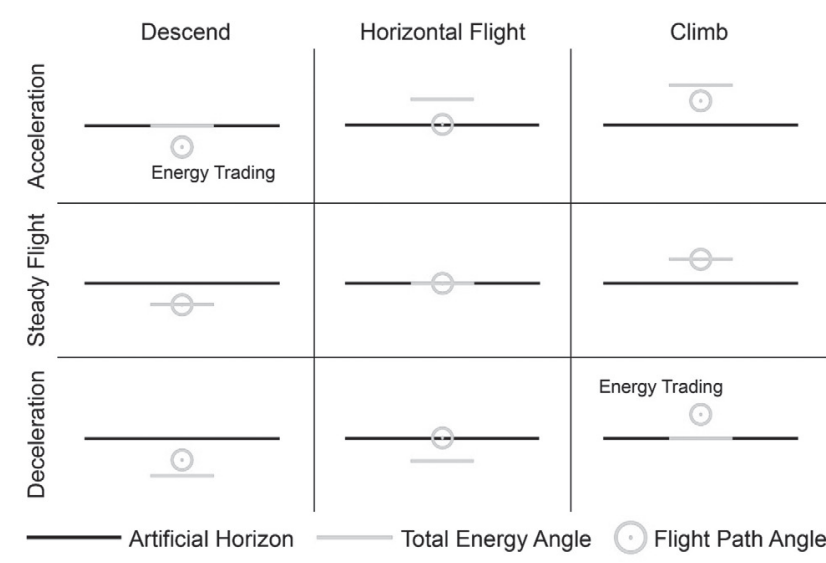

Figure 2. Relationship between total energy angle, flight path angle, and artificial horizon. "nxControl instead of pitch-andpower: A concept for enhanced manual flight control" by S. Müller, K. Schreiter, D. Manzey, \& R. Luckner (2016). CEAS Aeronautical Journal, 7, 110.

attentional demands when processing complex information derived from different parameters (Wickens, Hollands, Banbury, \& Parasuraman, 2013). Integrating this information in the PFD also serves the so-called proximity compatibility principle introduced by Wickens and Carswell (1995). That is, all primary flight parameters needed to aviate safely are presented along with information about the relative distribution of kinetic and potential energy in close spatial proximity.

It is expected that providing such augmented energy status information results in maintaining better energy awareness during manual flying, even when overall workload is high and flight patterns require complex energy adjustments. The first evidence for these proposed beneficial effects of energy status displays is provided by simulator studies suggesting that providing energy status information indeed improves the energy awareness of pilots and might reduce the cognitive burden of classical pitch-and-power flying (Catton et al., 2007; Müller et al., 2015; van den Hoven, de Jong, Borst, Mulder, \& van Paassen, 2010).

The second element of the nxControl system consists of an assistive flight controller for manual control of thrust and speed brakes (Müller et al., 2016). This controller, called nxController, directly uses the TEA as a command value to control the rate of change in total energy. For a given TEA setting, the controller executes the designated change in total energy by adjusting the needed thrust and speed brakes settings. At the same time, the controller compensates for the changes in thrust or drag induced by the aircraft configuration changes or atmospheric disturbances. In this way, a control concept is realized that directly corresponds to the format of augmented energy information in the energy displays referred to earlier. Beyond that, the general approach of a manual control augmentation is also compatible with the flight control laws implemented for sidesticks and pedals in current aircraft. Inputs to the controller are provided by a new nxLever, which replaces the conventional thrust lever. Combined with the new controller, a new nxStatus display is added to the cockpit's engine instrumentation, which provides feedback about the control inputs together with information about the current performance limitations of the aircraft. A detailed description of the nxController, the nxLever, and the nxStatus display, which is beyond the scope of this paper, can be found in the publication by Müller et al. (2016).

Recently, the first prototype of the complete nxControl system was developed and implemented in a fixed-base research simulator. In the first suitability study, 11 pilots had to perform four basic air work flight tasks with the new system. The results provided evidence of its feasibility. Specifically, it was shown that the participating pilots were able to perform the air work tasks with sufficient precision and less workload in terms of thrust lever movements after only a short familiarization with the new system.

The current study directly capitalizes on this research and intends to evaluate the consequences of human performance with the new system in a complex and demanding flight scenario. Experienced airline pilots with Airbus-type ratings had to manually fly a complex approach pattern with high demands on energy management in a fixed-base flight simulator. The pilots' performance, workload, and situation awareness (SA) during the approach were compared in conditions where pilots were and were not supported by different components of the new system. In order to separate possible effects of the energy display and the new controller, three different conditions were compared, including manual raw-data flying, manual flying with the enriched energyPFD, and manual flying with the complete nxControl system.

We hypothesized that flying with the nxControl system would lead to beneficial effects on performance, workload, and SA compared with conventional raw-data flying. Specifically, we expected that flying with the support of the nxControl system (a) would enable a higher precision in controlling airspeed and vertical flight path, (b) would lower the cognitive and physical effort involved in proper thrust control, and (c) would enhance the situation awareness of pilots with respect to energy-relevant flight parameters. It was further expected that the beneficial effects of the complete nxControl system, that is, the augmented display of energy status information in the PFD combined with the new concept of thrust control, would be greater than when adding only energy status information to the PFD. 


\section{Method}

\section{Participants}

In all, 24 licensed commercial airline pilots (all male, 10 captains, 14 first officers) were recruited as participants. Their average age was 40 years $(S D=12.6$ years) and ranged from 24 to 63 years. The pilots had flight experience of between 600 and 25,000 flight hours ( $M=8,505 \mathrm{hr}$, $S D=7,422 \mathrm{hr}$ ). All pilots possessed an Airbus type-rating (20 A320, 3 A330/A340, 1 A380). Therefore, they were familiar with the Airbus displays, sidestick, and its control laws. The pilots were offered an overall expense allowance for participation and access.

\section{Apparatus}

The study took place in a fixed-base flight simulator (Simulator for Educational Projects and Highly Innovative Research, SEPHIR) of the Chair of Flight Mechanics, Flight Control and Aeroelasticity of the Technische Universität Berlin. The simulator was configured as a simulation of the VFW 614-ATD and contained sidesticks with a manual flight control system. The flight characteristics, handling, and the cockpit layout of the simulation used were comparable to those of an Airbus A320. The outside view was simulated by a collimated, high-quality visual system. Primary flight displays, navigation displays, and engine displays were presented on $10^{\prime \prime}$ displays with a resolution of $1,280 \times 1,024$ pixels. For this study, the usual thrust levers were replaced with a newly developed nxLever that is compatible to both executing thrust control in a conventional way (i.e., control of N1) as well as controlling thrust according to the nxControl concept assisted by the nxController.

\section{Task}

The flight task included a considerably complex flight scenario with respect to energy management. The participants had to fly the required navigation performance (RNP) approach pattern to Salzburg LOWS runway 33 from south-east (Austro Control $\mathrm{GmbH}, 2014)$. In order to increase the demands on precise flight path control, the required performance was adjusted from RNP 0.3 to RNP 0.1 . This results in tolerances of $\pm 100 \mathrm{ft}$ vertically and \pm 0.1 nautical mile horizontally. Additionally, there were steady 15 -knot crosswinds from $057^{\circ}$ (minimal turbulences) that sometimes acted as tailwind owing to the required heading changes in the middle of the approach. The flight scenario was designed for the use of speed brakes to some extent. The visibility range of the simulated outside view was reduced by fog, thus the runway was not visible until reaching $1,240 \mathrm{ft}$ above ground which is $100 \mathrm{ft}$ above minimum decision height.

For purposes of experimental control, the pilots were obliged to follow a predefined approach procedure. This should ensure that the data of all participants are comparable, thus reducing influences and inflation of error variance due to different approach strategies. The prescribed procedure precisely defined when to configure flaps or landing gear by reference to the distance to next waypoint. In addition, required changes in airspeed were predefined as well. Thereby, the sequence was aligned to standard procedures in civil aviation, but altered in some critical points to enhance the observability of how well the participants would cope with energy adjustments.

\section{Design}

Three experimental conditions were compared in a withinsubject study design. In the first condition (conventional), the simulator was configured for conventional, manual raw-data flight without any enhanced energy information and no assistance of thrust control. This condition served as a control condition for assessing effects of the new nxControl components. The second condition (energyPFD) involved the conventional concept of thrust control but combined it with the energyPFD, that is, a PFD enriched by the presentation of the TEA and FPA (see Figure 1). Finally, the third condition ( $n x$ Control) consisted of all component of the nxControl system (energyPFD, the internal nxController for thrust and speed brakes control, and the nxStatus display). The sequence of experimental conditions was counterbalanced across participants.

\section{Procedure}

The experimental procedure was split into two parts. The first part served as an accommodation and training phase. It involved the pilot's familiarization with the simulator, the enhanced energy information in the PFD, and the nxControl system with nxStatus display. The familiarization included a standardized briefing and demonstrations of the meaning and functionality of the displays and systems. Afterward, the participants were trained on the basis of four standard flight tasks, such as air work and straight-in instrument landing system approaches. This familiarization was followed by an introduction and training of the RNP Salzburg approach and the predefined approach procedure. The training included one approach with conventional simulator configuration. During this approach, reminders with respect to the procedure and feedback were provided by the experimenter. Overall, the 
accommodation and training phase took about $1.5 \mathrm{hr}$, and was followed by a short break of approximately $15 \mathrm{~min}$.

The experimental data were collected in the second part of the experiment. This part was divided into three experimental blocks. Each block represented one condition of this study. At the beginning of each block, the pilots first performed a short practice flight to get accustomed to the specific simulator configuration. Afterward, the participants completed the RNP approach twice in a row with the respective simulator configuration. During all flights, the pilots were instructed to maintain the given flight path, airspeeds, and configurations as precisely as possible. They performed the approaches as pilot flying from the left seat. The experimenter served as pilot monitoring from the right seat and executed the ordered configuration changes of the aircraft, or selected the requested flight parameters, for example, altitude, speed, and heading. In addition, the experimenter mentioned whether flight parameters exceeded tolerances and read back the common callouts, too. After the three experimental blocks, a debriefing interview took place, in order to get additional information about the subjective assessment of the pilots concerning the different components of the nxControl system.

\section{Dependent Measures}

\section{Performance}

In order to assess how precisely the participants followed the prescribed approach pattern, deviations from the given reference pattern across time were examined by means of the root mean square error (RMSE) with reference to the prescribed altitude, airspeed, and lateral flight path targets. The precision to match the given target parameters of altitude and airspeed was particularly used for assessing the pilot's energy management performance. Both of these parameters are directly affected by the total energy and its distribution in manual flight. The lateral deviation from the requested flight path was additionally assessed in order to explore potential indirect impacts of the enhanced energy information or the nxControl system on flight path control.

\section{Workload}

Workload was assessed both subjectively and objectively. As an objective measure of workload involved in energy management, the input activity at the thrust and speed brake lever or nxLever was assessed, respectively. This input directly reflects the demands on a pilot in terms of the number of necessary thrust adjustments, that is, indicates how often the pilot has to invest cognitive and physical effort to re-assess and adjust the energy state of the aircraft. A lever movement was counted if a change in lever position was greater than $0.2 \mathrm{~cm}$ (approximately $0.5 \%$ of the entire lever range) in a time span of $2 \mathrm{~s}$.
The accumulated time span in which a lever movement was detected was related to the overall duration of the flight scenario. The higher the percentage, the more movement on the lever was required to complete the flight task, and thus, the higher the workload involved in energy management.

The NASA-TLX (Hart \& Staveland, 1988) was used to assess the subjectively experienced workload. Pilots provide their ratings on the six subscales after each single approach, that is, twice per condition. Owing to time constraints and methodological considerations, the NASA-TXL was used without the weighting procedure (Byers, Bittner, \& Hill, 1989).

\section{Situation Awareness}

An adjusted form of the Situation Awareness Global Assessment Technique (SAGAT) was used to objectively assess the pilot's SA while flying in each experimental condition (Endsley, 1988). In each experimental block, the simulation was suddenly frozen at one of three possible predefined points of the first approach. At the same time, the flight displays were blanked. The participants were then asked to recall the following eight flight parameters: indicated airspeed, lateral RNP deviation, vertical RNP deviation, barometric altitude, vertical speed, pitch, fan rotation speed N1, and heading. If the answers matched the actual parameter within a given range (see Table 1), it was counted as correct. The sum of all correct answers was then taken as an indicator of the level of SA varying between 0 and 8 . The specific point where the SAGAT assessment took place during the approach was counterbalanced across the experimental conditions.

In addition, a subjective SA assessment was performed by means of the SA-SWORD. This technique is an SA adaption of the Subjective Workload Dominance (SWORD) tool described by Vidulich, Ward, and Schueren (1991). The participants were required to judge which simulator configuration supported their SA better in three pair-wise comparisons. The judgments derived from the pairwise comparison were then checked for consistency and transformed into SA rating scores for each of the three configurations according to the procedure described by Vidulich et al. (1991). These scores describe the extent of subjectively perceived support of SA by the different configurations on a common scale.

\section{Data Analysis}

All statistical analyses, except analyses of SA assessments, were performed based on aggregated data from both approaches per experimental block. Differences between the three experimental conditions were statistically analyzed by means of a one-factorial analysis of variance 
Table 1. Tolerances for SAGAT evaluation

\begin{tabular}{lc}
\hline Flight parameter & Tolerance \\
\hline Indicated airspeed & $\pm 2.5 \mathrm{knots}$ \\
Lateral RNP deviation & $\pm 0.25 \mathrm{dots}$ \\
Vertical RNP deviation & $\pm 0.25 \mathrm{dots}$ \\
Altitude & $\pm 50 \mathrm{ft}$ \\
Vertical speed & $\pm 50 \mathrm{ft} / \mathrm{min}$ \\
Pitch & $\pm 1^{\circ}$ \\
Engine speed N1 & $\pm 2.5 \%$ \\
Heading & $\pm 2.5^{\circ}$ \\
\hline
\end{tabular}

Notes. This table shows the tolerances used to evaluate each flight parameter in the SAGAT questioning. If the participant's answers matched the actual parameter within the tolerance, it was counted as correct. The range of tolerance was derived from usual flight tolerances and readoff accuracy. RNP = required navigation performance. SAGAT = Situation Awareness Global Assessment Technique.

(ANOVA) with repeated measures. In the case of violations of the assumption of sphericity, a correction of degrees of freedom according to Huynh-Feldt was applied. Specific post hoc comparisons between means were conducted according to the Dunn-Šidák procedure for multiple comparisons.

\section{Results}

\section{Performance}

\section{Airspeed}

The means of the RMSE of airspeed for the three experimental conditions are shown in Figure 3. As is evident, the pilots were better able to maintain the preset airspeeds in the condition energyPFD $(M=2.8$ knots $)$ and nxControl ( $M=2.8$ knots), compared with the conventional condition ( $M=3.5$ knots). In the ANOVA this was reflected in a significant main effect, $F(1.58,36.35)=12.02, p<.001$, $\eta_{p}^{2}=.343$. Pairwise post hoc comparisons of the three means revealed the differences between energyPFD and conventional condition, $p<.001$, as well as between nxControl and conventional condition, $p=.005$, as significant. No significant effect, however, emerged between energyPFD and nxControl, $p=.945$.

\section{Altitude}

Figure 4 shows the mean RMSE of altitude for each experimental condition. In contrast to our assumptions, the average error in altitude was higher in the nxControl condition compared with the other two conditions (conventional: $M=31.2 \mathrm{ft}$; energyPFD: $M=32.0 \mathrm{ft}$ ). However, the differences were only small and did not become statistically significant, $F(1.51,34.79)=1.98, p=.163$, $\eta_{\mathrm{p}}^{2}=.079$.

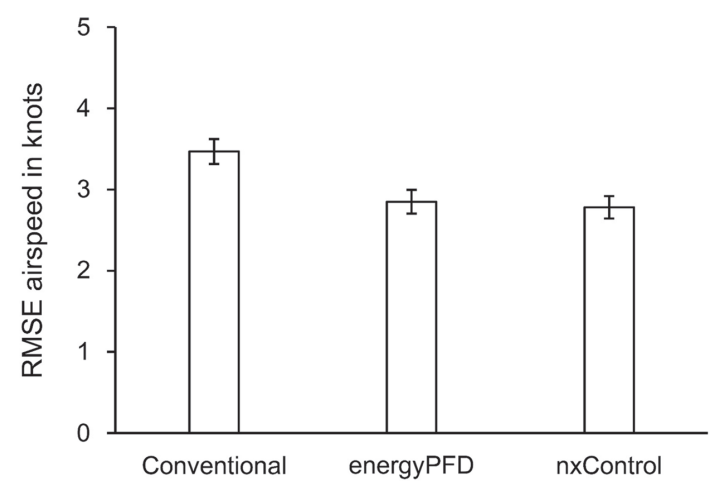

Figure 3. Mean RMSE of airspeed with standard error of each configuration. PFD = primary flight display.

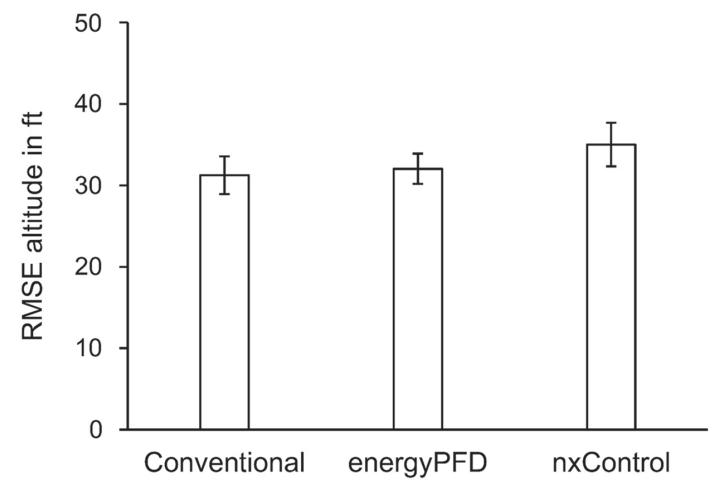

Figure 4. Mean RMSE of altitude with standard error of each configuration. PFD = primary flight display.

\section{Lateral Flight Path Deviation}

The mean lateral flight path deviations when flying with the three different configurations are shown in Figure 5. The mean deviations were the same for the conventional and energyPFD condition (0.030 nautical miles) and only slightly higher when flying with nxControl (0.035 nautical miles), with no statistically significant differences between conditions, $F(2,46)=2.00, p=.147, \eta_{p}^{2}=.080$.

\section{Workload}

\section{Lever Activity}

Figure 6 shows a graph of the lever activity assessment. The white bars mark the means of lever activity across the three experimental conditions. In the conventional and energyPFD conditions, they represent movements of the thrust lever, in the nxControl condition, it shows the movements of the nxLever.

The ANOVA comparing these conditions revealed that the pilots were able to perform the given flight task with lower lever activity in condition nxControl $(M=16.0 \%)$ as compared with both energyPFD $(M=24.4 \%)$ and conventional $(M=23.4 \%), F(1.35,31.09)=42.87$, 


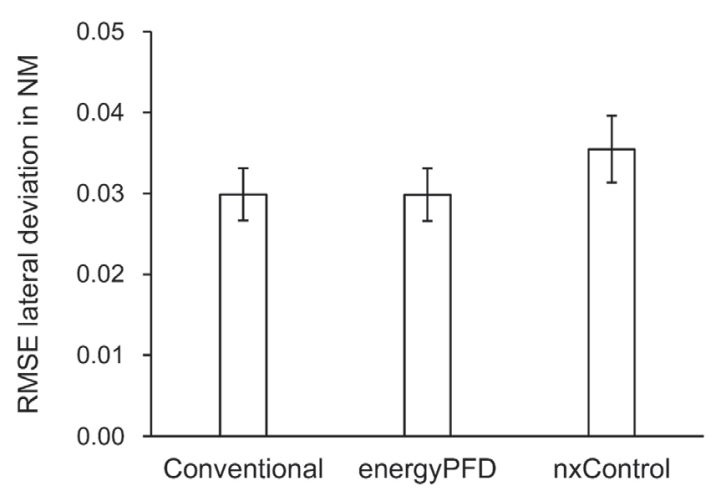

Figure 5. Mean RMSE of lateral flight path deviation with standard error of each configuration. $\mathrm{NM}=$ nautical miles. PFD = primary flight display.

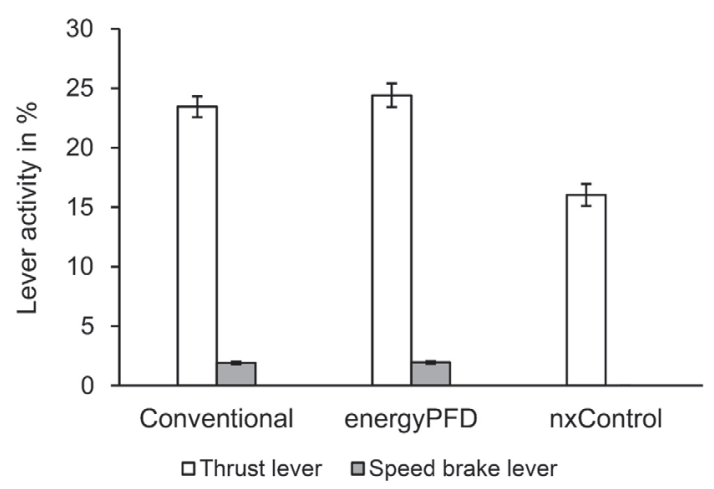

Figure 6. Means of lever activity with standard error of each configuration. White bars represent values for thrust lever or nxLever. Gray bars represent values for speed brake lever. PFD = primary flight display.

$p<.001, \eta_{\mathrm{p}}^{2}=.651$. Pairwise post hoc contrasts (Šidák) revealed that significantly less lever activity was needed in the condition nxControl compared with both other conditions (both $p<.001$ ), while no such effect emerged between conventional and energyPFD, $p=.299$.

The gray bars in Figure 6 represent the activity at the speed brake lever. Obviously, there are only data available in those conditions where speed brakes had to be operated by means of a separate input device, that is, conventional and energyPFD. As can be seen, no differences emerged between these two conditions with respect to this variable ( $M=1.9 \%$ for both conditions). In condition $\mathrm{nxControl}$, however, the speed brakes were automatically controlled by the nxController when activated by the pilot. Thus, no separate control inputs for speed brakes were necessary.

\section{NASA-TLX}

The data of the overall NASA-Task Load Index (TLX) score for condition energyPFD $(M=41.9)$ were slightly lower than in the conventional $(M=44.0)$ and nxControl ( $M=44.2)$ conditions. No significant difference emerged

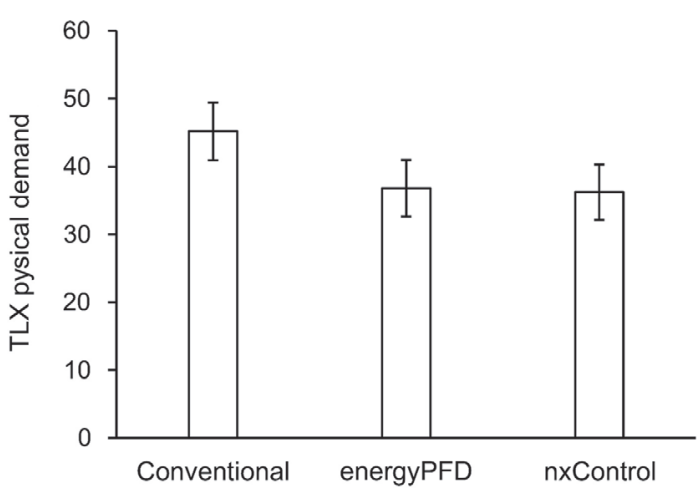

Figure 7. Mean TLX values of dimension physical demand with standard error of each configuration. PFD = primary flight display. TLX = Task Load Index.

between all three conditions, $F(2,46)=0.327, p=.723$, $\eta_{\mathrm{p}}^{2}=.014$. However, by looking at the specific workload dimensions, differences emerged with respect to the subjective assessment of physical demands (Figure 7). That is, the subjective ratings of the pilots' physical demand were about nine points lower for the conditions energyPFD $(M=36.8)$ and nxControl $(M=36.2)$ than the conventional condition $(M=45.2), F(2,46)=4.45, p=.017, \eta_{\mathrm{p}}^{2}=.162$. Pair-wise comparisons of the three means revealed a significant effect between the conventional and energyPFD conditions, $p=.029$. The difference between conventional and nxControl just missed reaching the usual level of significance, $p=.062$.

\section{Situation Awareness}

\section{SAGAT}

The mean numbers of correct answers (out of eight) in the SAGAT were 3.1 for the conventional condition, 2.9 for the energyPFD condition, and 2.5 for the nxControl condition. No significant effects emerged between the three conditions, $F(2,46)=1.307, p=.280, \eta_{p}^{2}=.054$.

\section{SA-SWORD}

The subjective ratings of pilots' SA as assessed by the SA-SWORD questionnaire are depicted in Figure 8. Before analyzing the data, the pair-wise ratings needed to be checked for consistency according to Crawford and Williams (1985). If the consistency measure exceeds $S^{2}=0.56$, the rating is discarded (Budescu, Zwick, \& Rapoport, 1986). Thus, 11 ratings were excluded from the analysis.

SA ratings were higher in the conditions energyPFD $(M=.61)$ and nxControl $(M=.61)$ than the conventional condition $(M=.17)$. The ANOVA proved this effect to be significant, $F(1.16,13.92)=7.06, p=.016, \eta_{p}^{2}=.370$. The post hoc analysis revealed significant effects between 


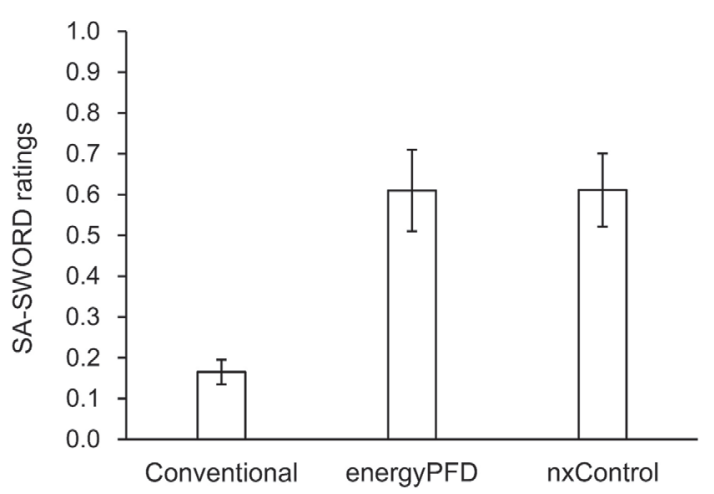

Figure 8. Mean SA-SWORD ratings with standard error of each configuration. PFD = primary flight display. SA-SWORD = Situation Awareness-Subjective Workload Dominance.

the conventional and nxControl conditions, $p=.001$, as well as between the conventional and energyPFD condition, $p=.006$. No significant effect was observable between energyPFD and nxControl, $p=1.00$.

\section{Discussion}

The present study investigated the human performance consequences of an energy-enhanced flight display and a newly proposed concept of total energy-related thrust controller on pilots' performance, workload, and SA. It was expected that the new system elements would increase performance and overall SA while at the same time reducing the pilots' workload. The results provide support for most of these assumptions.

Considering the effects on the different aspects of pilots' flying performance, especially regarding the results for airspeed, altitude, and flight path deviations, it is evident that the assumed performance benefits are particularly observable in the better matching of the airspeed requirements. However, no comparable effect was found for the altitude measure. With respect to matching the altitude requirements, the pilots achieved similar precision in all experimental conditions. This contrasts our expectations and is somewhat surprising, given that the vertical speed of an aircraft is directly affected by the management of energies in manual flight. Obviously, neither the provision of augmented energy information in the PFD nor the additional provision of the new assistive system for thrust control enabled the pilots to improve the precision of their vertical flight path control. One straightforward explanation of this lack of effect lies in the already very good performance of altitude or vertical speed control in the conventional condition. Thus, the lack of effects might not reflect a lack of effectiveness of the new system components but instead be related to a floor effect that has hindered finding additional improvements in performance. Two factors might have contributed to this effect. Firstly, maintaining the requested altitude within the tolerances of RNP 0.1 might not have been challenging enough to produce distinguishable results. However, RNP 0.1 requirements currently represent one of the most challenging requirements with respect to flight path precision and it seems highly implausible that they were too easy for our pilots. More likely, the already very good performance in the conventional condition might be explained by the control laws of the sidestick that, in accordance with the Airbus philosophy, were designed to hold the selected flight path. Thus, changes in thrust or drag cause changes in speed rather than changes in altitude. This might have biased the effects in our study with respect to airspeed rather than altitude. Neither effect was found for the precision of lateral flight path. However, controlling the lateral flight task is not directly connected to issues of energy management. In the current study this aspect of performance was simply assessed as a type of control variable to check for general changes in flight precision due to unspecific effects not directly related to the particular differences between experimental conditions.

Workload was assessed as lever activity and in terms of the subjective measure NASA-TLX. With respect to the effort needed for proper thrust control, that is, lever activity, the results suggest that nxControl can reduce the effort of pilots during energy management, but only with the whole system enabled, comprising the complete user interface as well as the nxController. These results validate outcomes of an earlier study that assessed nxControl in air work flight tasks and straight-in approaches, but originated only from a small amount of data (Müller et al., 2015, 2016). On a subjective level, this effect did not result in a perceived reduction of overall workload. No difference was found in the mean TLX ratings for the three conditions. Only a reduction of the experienced physical load was found which, however, emerged in both the energyPFD and nxControl conditions. It might be that the general demands of the complex approach dominated the subjective workload and masked any smaller differences between the experimental conditions.

The results of SA assessment were somewhat inconsistent. On the one hand, SAGAT did not show any significant differences between the SA assessed across conditions. It is worth mentioning that the SA score was generally very low in all experimental conditions. This might be due to a too narrowly chosen tolerance range that defines an answer as correct, or that in this specific flight situation some of the flight parameters are not crucial even in raw-data flight. On the other hand, the subjective rating of the SA-SWORD showed improvements of SA for the conditions energyPFD 
and nxControl. This proves that the pilots appreciated the additional information as an important improvement of their situation assessment. Yet, it cannot be excluded on the basis of the current data that this result might also reflect the consequence of pilots thinking that providing more information in principle implies by definition a better SA.

Overall, the results of the present study confirm the results of our last study that showed beneficial human performance effects associated with augmented energy information and a new logic of thrust control (Müller et al., 2016). It is striking that these effects again emerged even though the participating pilots only had limited practice with the new system components and their subsequent performance was comparable to a condition (conventional raw-data flying) in which they had much more training. This suggests that the new system components are easy to understand and to apply. It also suggests that the findings of the present study are somewhat conservative and even more beneficial effects might be expected if pilots gain more experience with the new components.

The results also provide additional insights on the specific effects of energy-enhanced displays and the new concept of thrust control. Most of the beneficial performance effects, with the exception of the effects on lever activity, were already found in the energyPFD condition where the pilots used the conventional concept of thrust control but were supported by an energy display added to the PFD. This provides direct empirical evidence for the presumed advantages of providing augmented energy information to pilots (e.g., Amelink et al., 2005; Lambregts et al., 2008) and supports our expectation that providing this information in the form of an emergent figure has made it easier for the pilots to maintain a proper distribution of energy and target flight states. It further complements previous findings indicating that energy displays can improve a pilot's energy management reflected in better precision of flying (van den Hoven et al., 2010). Specifically, the current study proves this effect is valid also in the context of a complex flight scenario when energy information is added to the standard head-down instrumentation. However, the beneficial effects of a more precise adjustment of total energy reflected in a reduced number of lever movements were only gained by using the complete nxControl system, comprising the energy-enhanced PFD, nxController, nxLever, and nxStatus display. Since each adjustment of thrust can be considered as an output of a new cognitive assessment of the current pitch-and-power relationship, the finding of reduced lever movements confirms our expectation that the proposed nxControl approach indeed can unload the pilot from the demands of pitch-and-power flying to some extent. At the same time it also can reduce the physical load involved in lever activity to control thrust.

In conclusion, the results of the current study suggest that the proposed approach of nxControl as an alternative to conventional thrust control can effectively and better support manual flying, which need to be maintained and applied in order to maintain pilots' competency to always take over manual control of their aircraft. Providing such support will further enable pilots to fly manually with sufficient precision even in future aviation with presumably more complex and demanding flight trajectories.

\section{Acknowledgments}

This work is funded by the DFG (German Research Foundation) under Contract LU 1397/3-1, MA 3759/3-1. The authors thank all pilots who participated in the experiments and Vereinigung Cockpit e.V. that supported the experiment by distributing the invitation to their members. Further thanks are due to B. Boche, D. Bieniek, A. Kaden, I. Karakaya, and M. Schaumburg for their support at different phases of this research.

\section{References}

Amelink, M. H., Mulder, M., van Paassen, M. M., \& Flach, J. (2005). Theoretical foundations for a total energy-based perspective flight-path display. The International Journal of Aviation Psychology, 15(3), 205-231. doi: 10.1207/s15327108ijap1503

Austro Control GmbH. (2014). Instrument approach chart - ICAO (RNAV (RNP) Z RWY 33). Retrieved from http://eaip.austrocontrol. at/lo/150430/Charts/LOWS/LO_AD_2_LOWS_24-6-6_en.pdf

Bennet, K. B., \& Flach, J. M. (2011). Display and interface design: Subtle science, exact art. Boca Raton, FL: CRC Press.

Budescu, D. V., Zwick, R., \& Rapoport, A. (1986). A comparison of the eigenvalue method and the geometric mean procedure for ratio scaling. Applied Psychological Measurement, 10(1), 69-78. doi: 10.1177/014662168601000106

Byers, J. C., Bittner, A. C., \& Hill, S. G. (1989). Traditional and raw task load index (TLX) correlations: Are paired comparisons necessary? In A. Mital (Ed.), Advances in industrial ergonomics and safety (pp. 481-485). Cincinnatio, OH: Taylor \& Francis.

Catton, L., Starr, A., Noyes, J. M., Fisher, A., \& Tim, P. (2007). Designing energy display formats for civil aircraft: Reply to Amelink, Mulder, van Paassen, and Flach. The International Journal of Aviation Psychology, 17(1), 31-40.

Crawford, G., \& Williams, C. (1985). The analysis of subjective judgment matrices. Tech. rep. Santa Monica, CA: United States Air Force.

Dutch Safety Board. (2010). Crashed during approach, Boeing 737-800, near Amsterdam Schiphol Airport, 25 February 2009. Final report of Dutch Safety Board.

Endsley, M. R. (1988). Situation awareness global assessment technique (SAGAT). In Proceedings of the IEEE National Aerospace and Electronics Conference (pp. 789-795). New York, NY: IEEE.

Federal Aviation Administration. (2013). Safety alert for operators: Manual flight operations (SAFO 13002). Washington, DC: US Department of Transportation. 
Federal Aviation Administration. (2016). Enhanced FAA oversight could reduce hazards associated with increased use of flight deck automation (Report No. AV-2016-013). Washington, DC: US Department of Transportation.

Filippone, A. (2012). Advanced aircraft flight performance. Cambridge, UK: Cambridge University Press.

Hart, S. G., \& Staveland, L. E. (1988). Development of NASA-TLX (Task Load Index): Results of empirical and theoretical research. In P. A. Hancock \& N. Meshkati (Eds.), Human mental workload (pp. 139-183). Amsterdam, The Netherlands: North Holland Press.

Lambregts, T., Rademarker, R., \& Theunissen, E. (2008). A new ecological primary flight display concept. In Digital Avionics Systems Conference (pp. 4.A.1-1-4.A.1-20). St. Paul, MN: IEEE. doi: 10.1109/DASC.2008.4702820

Müller, S., Manzey, D., Schreiter, K., \& Luckner, R. (2015). Implementing energy status in head down cockpit displays: Impact of augmented energy Information on pilot's performance. In Proceedings of the Human Factors and Ergonomics Society 59th Annual Meeting (pp. 926-930). Santa Monica, CA HFES. doi: 10.1177/1541931215591270

Müller, S., Schreiter, K., Manzey, D., \& Luckner, R. (2016). $\mathrm{n} \times$ Control instead of pitch-and-power: A concept for enhanced manual flight control. CEAS Aeronautical Journal, 7(1), 107-119. doi: 10.1007/s13272-016-0182-7

National Transportation Safety Board (Producer). (2013). Chairman Hersman's final media briefing on Asiana flight 214 crash July 11, 2013 [Video file]. Available from http://www. youtube.com/watch?v=|1GopE_sivY

van den Hoven, M., de Jong, P. M., Borst, C., Mulder, M., \& van Paassen, M. M. (2010). Investigation of energy management during approach-Evaluating the total energy-based perspective flight-path display. In AIAA Guidance, Navigation, and Control Conference (pp. 1-21). Reston, VA: AIAA.

Vidulich, M. A., Ward, F. G., \& Schueren, J. (1991). Using the Subjective Workload Dominance (SWORD) technique for projective workload assessment. Human Factors, 33(6), 677-691.

Wickens, C. D., \& Carswell, C. (1995). The proximity compatibility principle: Its psychological foundation and relevance to display design. Human Factors, 37(3), 473-494.

Wickens, C. D., Hollands, J. G., Banbury, S., \& Parasuraman, R. (2013). Engineering psychology and human performance (4th ed.). Boston, MA: Pearson. 